Research Article

\title{
Enhancing Human-Computer Interaction in Digital Repositories through a MCDA-Based Recommender System
}

\author{
Christos Troussas (iD, Akrivi Krouska $(\mathbb{D}$, and Cleo Sgouropoulou \\ University of West Attica, Egaleo, Greece \\ Correspondence should be addressed to Christos Troussas; ctrouss@uniwa.gr
}

Received 29 April 2021; Revised 6 September 2021; Accepted 6 October 2021; Published 19 October 2021

Academic Editor: Marco Porta

Copyright (C) 2021 Christos Troussas et al. This is an open access article distributed under the Creative Commons Attribution License, which permits unrestricted use, distribution, and reproduction in any medium, provided the original work is properly cited.

\begin{abstract}
Digital repositories contain a large amount of content, which is available to heterogeneous groups of people. As such, in many cases people encounter difficulties in finding specific content which is related to their preferences. In view of this compelling need and towards advancing human-computer interaction, this paper presents a recommender system which is incorporated in a digital repository. The recommender system is designed using multiple-criteria decision analysis (MCDA) and more specifically the weighted sum model (WSM) in order to refine the delivered content to the users. It also considers several users' characteristics (their preferences as depicted by the content they visited or searched and by the frequency of searches/visits) and features of the content (content types and traffic). The recommender system outputs the suggestions of content to users based on their preferences and interests. The presented recommender system was evaluated by real users, and the results show a high degree of accuracy in the recommended content and satisfaction by users.
\end{abstract}

\section{Introduction}

Human-computer interaction (HCI) explores the design and use of computer technologies, targeting the interfaces between computers and their users (people). Researchers in the field of HCI study the means through which people interact with computers and develop technologies that, in essence, help humans interact with them in an effective way $[1,2]$.

As a research topic, HCI is a confluence of computer science and several other fields of study, such as design, media studies, behavioral sciences, and so on. The term involves multiple applications for a computer, and this happens as an open-ended conversation between the user and the computer. The definition of this interconnection contrasts human-computer interaction with human-tohuman interaction, a comparison that is significant for the field's theoretical considerations.

In view of the above, $\mathrm{HCI}$ is a significant field of research that should be respected in the design and development of several systems and applications [3], such as digital repositories. A digital repository is a digital information management and storage mechanism [4]. Repositories may, in their emphasis, be subject or institutional. Putting material into an institutional repository allows it to be handled and maintained by organizations and employees and hence to gain full benefit from it. Study, learning, and administrative processes may be assisted by a repository. Repositories use open practices to make sure that it is easy to access the content they contain in order to trace and recover it for further use. By using these internationally accepted principles, frameworks which import, export, classify, store, and retrieve digital content within the repository can be developed.

For a variety of purposes and users, digital repositories may contain a wide volume of content. Currently, what is included in a repository is less of a technical or software capacity problem and more of a policy option taken by each organization or administrator. Study outputs, such as journal articles or research data, e-theses, e-learning objects, and instructional materials, and administrative data may actually contain content. Some repositories take only certain objects (such as theses or journal papers), whereas others aim to compile all credible research work created by the institution, constrained only by the retained rights of 
publishers by each author. Several other sophisticated objects, though, raise a technical challenge (websites, advanced learning objects, 3D topographical representations, other datasets, and so on).

Recommender systems are mechanisms for applications intended to provide users with advice [5]. These guidelines are meant to make decision making simpler for people in a variety of areas where information filtering is important. For instance, they are used in entertainment applications for film, songs, etc., in recommendations for travel and restaurant web services, in video or post recommendations on social networks, in e-learning applications to suggest teaching activities or user projects, etc. Therefore, by making users find the content they are interested in easily and effectively, such programs can solve the issue of overinformation.

As such, recommender systems can be integrated in digital repositories in order to enhance the interaction between the users and the computers and help them to have a more personalized experience [6]. In digital repositories, recommender systems can offer suggestions about the content to be delivered, and thus it can be more tailored to the needs and preferences of the users.

To build a mechanism that delivers adequate recommendations to users of digital repositories is a difficult process that requires the recognition and checking of different features concerning the preferences of users, e.g., what they have visited or searched, search frequency, etc. Hence, the utilization of multiple-criteria decision analysis (MCDA) techniques seems to be the optimal solution for making decisions about the content to be delivered to the users of digital repositories. In decision making, MCDA specifically evaluates several competing factors. In our approach, the weighted sum model (WSM) is used. The explanation for this selection is that in terms of a variety of decision parameters, WSM is the most renowned and easiest MCDA approach for testing a variety of choices [7]. WSM uses several gain metrics for weight and estimates the significance of each option.

In the related scientific literature, there have been several research works that explore the development of recommender systems in different domains, e.g., digital repositories [6, 8-12], entertainment web portals [13-16], digital education [17-20], and social settings [21-24]. In the aforementioned research studies, the algorithmic approaches that have been mainly adopted are content-based filtering, stereotypes and model-based approaches, collaborative filtering, and hybrid techniques. A recent study of 2020 [25] notes that such methods (e.g., collaborative filtering) present several drawbacks, like the cold start problem, which means that the system cannot make any inferences for users/items about whom/which information have not been gathered in a sufficient degree. Such problems may be overcome with the use of the MCDA method. Indeed, MCDA has been used in recommender systems for refining the suggestion of content to users [26-28], but to the best of our knowledge, it has not been applied yet sufficiently in digital repositories. In a review work of 2019 [29] about recommender systems for digital repositories, the author confirms that the main algorithms used by such systems are mainly the above ones, including content-based and collaborative filtering and hybrid methods. The same work underlines that more adequate recommender systems in digital repositories can be powered by current approaches of information retrieval, e.g., MCDA and WSM.

In view of the above, this paper presents a recommender system which is incorporated in a digital repository. The digital repository includes assignments of undergraduate computer science students as well as learning material for computer science courses. The recommender system is designed using MCDA and more specifically WSM in order to refine the delivered content to the users. It also considers several users' characteristics, namely, their preferences as depicted by the content they visited or searched and by the frequency of searches/visits, and features of the content, namely, content types and traffic. The recommender system outputs the suggestions of content to users based on their preferences and interests. The novelty and main contribution of this paper is the employment of MCDA, and specifically WSM, for digital repositories, which is considered a novel way for recommendation in this context. In addition, the inputs of the recommender module are characteristics (e.g., content traffic and type, frequency of searches and visits, etc.) which seem to be powerful for the recommendation of digital repositories' units. The presented recommender system was evaluated by real users, and the results show a high degree of accuracy in the recommended content and satisfaction by users.

\section{MCDA-Based Recommender Module: Architecture and Description}

In order for the system to provide proper content to users, it takes as input both user's preferences for enhancing its individualization and content features for enhancing its quality and appropriateness. As such, the input of the recommender module includes the following:

(i) What a user has visited: the content that a user visits is an important characteristic since it depicts his/her preferences. For example, if the content is an article in computer science, then it is assumed that the user is interested in technology.

(ii) What a user has searched: the content that a user has searched concerns actually the filters she/he has used to refine the content. More specifically, specific information concerning the searched items can be gathered.

(iii) Frequency of searches/visits: the number of times a user searches for specific content or visits it can be of great importance since it depicts his/her preferences.

(iv) Type of content: the type of content concerns the domain for which it has been created.

(v) Content traffic: the content traffic declares the number of times each content has been visited. 
Based on these log files, the MCDA-based technique is applied for delivering personalized content to users. The logical architecture of the recommender module is presented in Figure 1.

Recommendation techniques are essential for helping users in a digital community to find information that is more relevant to their interests and needs. Assuming a set of users $U$ and a set of content $C$ that can be recommended to them, the goal of a recommender system is for each user $u \in U$ to estimate the utility function $R(u, c)$ for each content $c \in C$ and then to choose the subset of content $C$ that maximizes $R$, which will be the recommended content RC, i.e.:

$$
\forall u \in U, R C=\arg \max R(u, c), \quad \text { where } c \in C .
$$

The most common technique used by recommender systems is collaborative filtering, where the tastes and likings of similar users are exploited when formulating recommendations for a particular user [30]. However, this method considers only user reviews, having limited ability to recommend when no reviews exist or a user is new or new releases occur. The data sparsity affects negatively similarity information due to few or no co-rated items between two users [30]. As such, for improving the quality and the accuracy of the recommendations, MCDA is one of the most well-known practices with excellent results in this field $[15,27-29]$. Using this method, the recommender system is able to represent more complex preferences of each user, since it takes into consideration multiple criteria for making its suggestions.

WSM is a MCDA method used in this work, in which there are a range of alternatives and the best ones are determined based on multiple criteria assigning a weight to each one. In particular, when a user searches for content in the digital repository, the presented recommendation system firstly selects a subset of its digital items based on the search filters. However, the critical issue is to order properly the returned items according to user's preferences and content's quality. Therefore, the following values are calculated for each item in the subset based on the system log files:

(i) Values concerning users' actions:

(a) VC: the degree that the item is relevant to the content visited by the user, ranging from 0 (irrelevant) to 1 (it has been visited).

(b) SC: the degree that the item is relevant to the content searched by the user, ranging from 0 (irrelevant) to 1 (it has been searched).

(c) FC: the frequency of searches and visits the user made for the specific item.

(ii) Values concerning item attributes:

(a) DC: the degree that the domain of the item is relevant to user's preferences, ranging from 0 (low) to 1 (high).

(b) CT: the normalized value of the number of times the item has been visited by the users, ranging from 0 (low) to 1 (high).

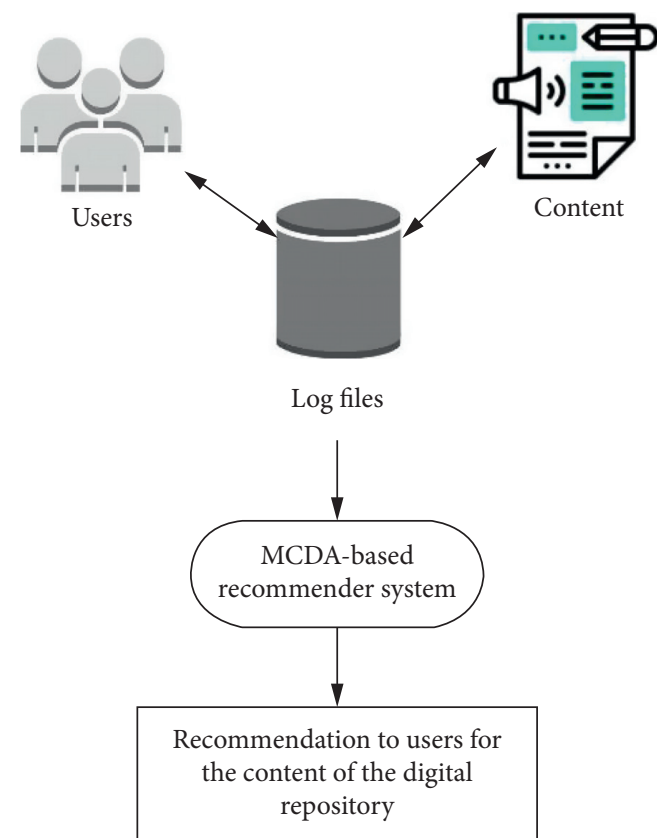

Figure 1: Logical architecture.

The aforementioned values are calculated using the metadata of each item (e.g., the relevance of the item with each subject category, the keywords of the item, etc.) in conjunction with the metadata of users (e.g., searched subject category, keywords used in searches, etc.). For all the metadata, a distance metric is applied to calculate the above values [31].

Regarding the weights of each criterion, they were determined by 10 experts (faculty members of computer science in Greek universities) through interviews based on the focus they want to give on the order of content. The weights can be adjusted properly according to the problem space. In our case, the experts decided that the relevance of item domain with users' preferences is of great importance, giving $\mathrm{DC}$ variable the highest weight $\left(w_{\mathrm{DC}}=0.3\right)$. The relevance between the item and the content visited by the user and the number of times the item has been visited by the users are followed with weight values $w_{\mathrm{VC}}=0.25$ and $w_{\mathrm{CT}}=0.2$, respectively. Finally, the experts assessed with lower weights the frequency of searches and visits the users made for the specific item and the relevance of the item with the content visited by the users, i.e., $w_{\mathrm{FC}}=0.15$ and $w_{\mathrm{SC}}=0.1$ correspondingly.

Figure 2 illustrates the recommendation process of the presented system. In particular, the steps can be described as follows:

(1) The user searches for content based on filters, such as keywords, subject categories, and so on.

(2) The system selects a subset of content, namely, RC.

(3) The system calculates the values of VC, SC, FC, DC, and CT for each content $c$ in RC. 


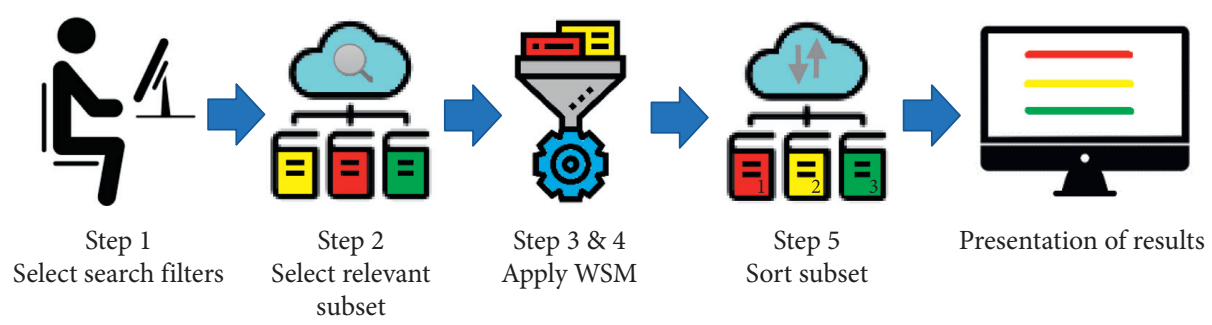

Figure 2: Algorithmic process for item recommendation.

(4) The system applies WSM to evaluate the utility function of content $c\left(c^{\text {WSM-score }}\right)$ for the particular user:

$$
\begin{aligned}
c^{\mathrm{WSM}-\text { score }}= & w_{\mathrm{VC}} * \mathrm{VC}+w_{\mathrm{SC}} * \mathrm{SC}+w_{\mathrm{FC}} * \mathrm{FC} \\
& +w_{\mathrm{DC}} * \mathrm{DC}+w_{\mathrm{CT}} * \mathrm{CT} .
\end{aligned}
$$

(5) The system sorts the content of RC based on $c^{\mathrm{WSM}-}$ score in descending order.

For a better understanding of the algorithm, an example of operation of the proposed recommender system utilizing the WSM technique is given in Table 1. In particular, assume that two users ( $u 1$ and $u 2$ ) with different profiles search for content based on the same filters and the content returned consists of 5 items. The system calculates the values of the variables concerned users' actions and item attributes, namely, VC, SC, FC, DC, and CT. Since the two users have different profiles, the values of all variables are different among them, except from CT which is independent of users' characteristics. Afterwards, the system calculates the WSM score for each item using the weights defined by the experts. These score values determine the order in which the items will be returned to the users. Therefore, according to the example of Table 1, the ranking for $u 1$ is $c 1, c 5, c 3, c 4$ and $c 2$, whereas, for $u 2$, it is $c 2, c 1, c 4, c 3$, and $c 5$.

\section{Evaluation Results and Discussion}

In order to maintain the usefulness and efficiency of a software application's capacities, evaluation plays a vital role. The key for developing qualitative applications is the assessment of the importance of a program by all its users. This section presents the evaluation of the presented recommender system which was integrated in a digital repository by real users. The population of the evaluation includes 100 users of a digital repository who are employees in a public Greek university. The population was separated in two equal groups (Group A and Group B). Group A used our MCDAbased recommender system incorporated in a digital repository while Group B used the digital repository and received suggestions based solely on their visits of content. The digital repository was created for the sake of this experiment and includes assignments of 500 university students, educational images and videos, and learning materials of computer science courses. Both groups used the repository for a period of three months. After that period, the users of both groups were asked to answer the following questions: (a) Rate the accuracy of the content delivered to you (Q1) (0-10).

(b) Rate how interesting the delivered content was (Q2) (0-10).

(c) Rate your satisfaction (Q3) (0-10).

(d) Rate your overall experience (Q4) (0-10).

The answers of Group A are aggregated and depicted in Figure 3. Analyzing the results, it is observed that there is a high rating of the accuracy of the content being delivered to users. A reason for this is because the system takes into consideration the users' preferences, such as the searches and visits of the users. Furthermore, the results show that the delivered content was interesting for the users. This is because the system considers the degree that the domain of the item is relevant to users' preferences. Also, the satisfaction as well as the overall experience of the users has been reported to be very high given that the system employs algorithmic techniques which offer personalization and adaptivity to users' profiles.

Furthermore, the answers of both groups were analyzed using the $t$-test. The alpha value was set equal to 0.05 , and the $p$ values were analyzed. Based on the results, for the null hypothesis: "There is no difference between the two groups of users," the $t$-test rejects the hypothesis for all the questions, meaning that there is statistical significance and the presented recommender system of Group A is more efficient than the one of Group B. The $t$-test results are shown in Table 2.

The aforementioned results were anticipated. Our approach employing MCDA for refining the recommendation of content in a digital repository is a novel technique that can further enhance the interaction between users and computers. Based on these results, it is shown that the accuracy of the recommendations is high as well as the delivered content is interesting for the users. Moreover, the overall users' satisfaction and experience, when interacting with the system, is high.

In the following, the log data of the two systems (which have been used by Groups A and B) are provided and compared to show the superiority of the presented novel approach (Table 3). In particular, the number of searches on a specific topic as well as the ranking of the items selected by the users was analyzed. The first factor refers to the average of the attempts the users made to obtain the desired results, while the second one refers to the possibility that the selected items belong to the first page of the algorithms' results, consisting of 10 items. Analyzing these log data, the searches on a specific topic that were made by Group A were fewer than the corresponding searches by Group B (2.6 and 5.3, 
TABLE 1: Example of operation.

\begin{tabular}{|c|c|c|c|c|c|c|c|c|c|c|c|c|c|c|}
\hline & \multicolumn{2}{|c|}{$\begin{array}{c}\mathrm{VC} \\
w_{\mathrm{VC}}=0.25\end{array}$} & \multicolumn{2}{|c|}{$\begin{array}{c}\mathrm{SC} \\
w_{\mathrm{SC}}=0.1\end{array}$} & \multicolumn{2}{|c|}{$\begin{array}{c}\mathrm{FC} \\
w_{\mathrm{FC}}=0.15\end{array}$} & \multicolumn{2}{|c|}{$\begin{array}{c}\mathrm{DC} \\
w_{\mathrm{DC}}=0.3\end{array}$} & \multicolumn{2}{|c|}{$\begin{array}{c}\mathrm{CT} \\
w_{\mathrm{CT}}=0.2\end{array}$} & \multicolumn{2}{|c|}{ WSM score } & \multicolumn{2}{|c|}{ Ranking } \\
\hline & $u 1$ & $u 2$ & $u 1$ & $u 2$ & $u 1$ & $u 2$ & $u 1$ & $u 2$ & $u 1$ & $u 2$ & $u 1$ & $u 2$ & $u 1$ & $u 2$ \\
\hline$c 1$ & 0.73 & 0.56 & 0.42 & 0.78 & 0.11 & 0.89 & 0.68 & 0.47 & 0.62 & 0.62 & 0.8125 & 0.6165 & 1 & 2 \\
\hline$c 2$ & 0.16 & 0.69 & 0.29 & 0.62 & 0.38 & 0.57 & 0.13 & 0.79 & 0.49 & 0.49 & 0.254 & 0.6550 & 5 & 1 \\
\hline$c 3$ & 0.58 & 0.23 & 0.91 & 0.17 & 0.69 & 0.32 & 0.48 & 0.12 & 0.81 & 0.81 & 0.6455 & 0.3205 & 3 & 4 \\
\hline$c 4$ & 0.36 & 0.44 & 0.55 & 0.12 & 0.47 & 0.51 & 0.24 & 0.56 & 0.72 & 0.72 & 0.4315 & 0.5105 & 4 & 3 \\
\hline$c 5$ & 0.87 & 0.31 & 0.63 & 0.37 & 0.72 & 0.23 & 0.81 & 0.13 & 0.34 & 0.34 & 0.6995 & 0.2560 & 2 & 5 \\
\hline
\end{tabular}

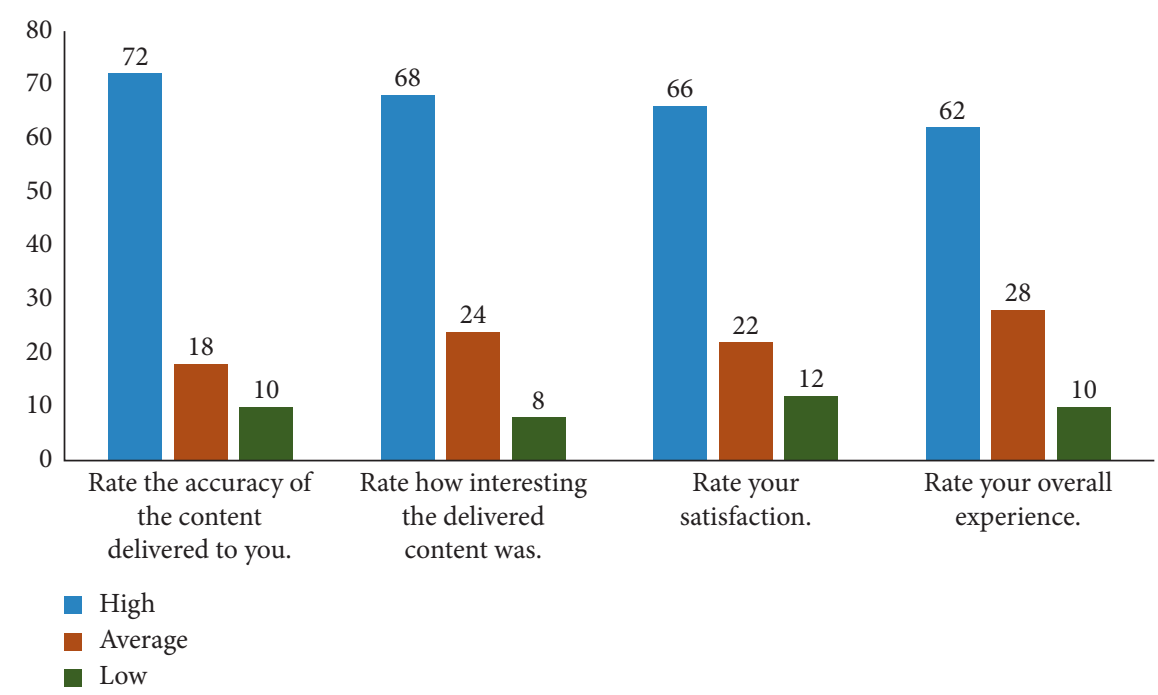

Figure 3: Answers of users.

TABle 2: Results of $t$-test.

\begin{tabular}{|c|c|c|c|c|c|c|c|c|}
\hline & \multicolumn{2}{|c|}{ Q1 } & \multicolumn{2}{|c|}{ Q2 } & \multicolumn{2}{|c|}{ Q3 } & \multicolumn{2}{|c|}{ Q4 } \\
\hline & Variable 1 & Variable 2 & Variable 1 & Variable 2 & Variable 1 & Variable 2 & Variable 1 & Variable 2 \\
\hline Mean & 8,32 & 7,3 & 7,84 & 6,88 & 7,94 & 6,76 & 8,54 & 7,1 \\
\hline Variance & 0,997551 & 0,867347 & 1,647347 & 2,189388 & 0,95551 & 1,206531 & 0,58 & 1,357143 \\
\hline Observations & 50 & 50 & 50 & 50 & 50 & 50 & 50 & 50 \\
\hline Hypothesized mean difference & \multicolumn{2}{|c|}{0} & \multicolumn{2}{|c|}{0} & \multicolumn{2}{|c|}{0} & \multicolumn{2}{|c|}{0} \\
\hline $\mathrm{df}$ & \multicolumn{2}{|c|}{98} & \multicolumn{2}{|c|}{96} & \multicolumn{2}{|c|}{97} & \multicolumn{2}{|c|}{84} \\
\hline$t$ Stat & \multicolumn{2}{|c|}{5,281504} & \multicolumn{2}{|c|}{3,465575} & \multicolumn{2}{|c|}{5,674598} & \multicolumn{2}{|c|}{7,315882} \\
\hline$p(T \leq t)$ one-tail & \multicolumn{2}{|c|}{$3,86 E-07$} & \multicolumn{2}{|c|}{0,000396} & \multicolumn{2}{|c|}{$7,21 E-08$} & \multicolumn{2}{|c|}{$6,96 E-11$} \\
\hline$t$ critical one-tail & \multicolumn{2}{|c|}{1,660551} & \multicolumn{2}{|c|}{1,660881} & \multicolumn{2}{|c|}{1,660715} & \multicolumn{2}{|c|}{1,663197} \\
\hline$p(T \leq t)$ two-tail & \multicolumn{2}{|c|}{$7,73 E-07$} & \multicolumn{2}{|c|}{0,000793} & \multicolumn{2}{|c|}{$1,44 E-07$} & \multicolumn{2}{|c|}{$1,39 E-10$} \\
\hline$t$ critical two-tail & \multicolumn{2}{|c|}{1,984467} & \multicolumn{2}{|c|}{1,984984} & \multicolumn{2}{|c|}{1,984723} & \multicolumn{2}{|c|}{1,98861} \\
\hline
\end{tabular}

Table 3: Analysis of log data.

\begin{tabular}{lcc}
\hline Population & Number of searches on a specific topic & Possibility of items selected by user appearing in first page (\%) \\
\hline Group A & 2.6 & 84 \\
Group B & 5.3 & 46 \\
\hline
\end{tabular}

respectively), indicating that the outputs of the presented technique were more relevant to the searches of Group A. It was observed that Group B made more searches, giving different keywords to refine the search and changing the display filters of the results in the effort to find more relevant items. Moreover, the percentage of the items in the first page of results that Group A visited was $84 \%$, higher than the corresponding one that Group B selected (46\%). This fact reflects the quality of results of the presented algorithm as well as its accurate ranking. It should be noted that Group B was browsing through the results' pages much more than Group A, searching for relevant items. 


\section{Conclusions}

This paper presents a novel framework for recommendations delivery which can enhance human-computer interaction in digital repositories. MCDA and, more specifically WSM, was employed to build the recommender system aiming to refine the delivered content to the users. The recommender system also considers several users' characteristics (their preferences as depicted by the content they visited or searched and by the frequency of searches/visits) and features of the content (content types and traffic). The recommender system outputs the suggestions of content to users based on their preferences and interests. The presented recommender system was evaluated by real users, and the results show a high degree of accuracy in the recommended content and satisfaction by users.

Our future work includes a more detailed evaluation in order to further assess the efficacy of our approach. Moreover, our future plan is to blend machine learning techniques with the WSM technique for exploring the amelioration of the recommendation process.

\section{Data Availability}

The data used to support the findings of this study have not been made available because they contain information that could compromise research participant privacy/consent.

\section{Conflicts of Interest}

The authors declare that they have no conflicts of interest.

\section{References}

[1] J. Tantiongloc, D. A. Mesa, R. Ma et al., “An information and control framework for optimizing user-compliant humancomputer interfaces," Proceedings of the IEEE, vol. 105, no. 2, pp. 273-285, 2017.

[2] C. Troussas, A. Krouska, and M. Virvou, "Adaptive e-learning interactions using dynamic clustering of learners' characteristics," in Proceedings of the 2019 10th International Conference on Information, Intelligence, Systems and Applications (IISA), pp. 1-7, Patras, Greece, July 2019.

[3] Y. Ji, Y. Yang, F. Shen, H. T. Shen, and X. Li, "A survey of human action analysis in HRI applications," IEEE Transactions on Circuits and Systems for Video Technology, vol. 30, no. 7, pp. 2114-2128, 2020.

[4] T. Crocken and A. Washington, "Strategies and tools for digital repository selection and migration," in Proceedings of the 2019 ACM/IEEE Joint Conference on Digital Libraries (JCDL), pp. 446-447, Champaign, IL, USA, June 2019.

[5] S. Amara and R. R. Subramanian, "Collaborating personalized recommender system and content-based recommender system using TextCorpus," in Proceedings of the 2020 6th International Conference on Advanced Computing and Communication Systems (ICACCS), pp. 105-109, Coimbatore, India, March 2020.

[6] B. Guan, L. Hu, P. Liu, H. Xu, Z. Fu, and Q. Wang, "dpSmart: a flexible group based recommendation framework for digital repository systems," in Proceedings of the 2019 IEEE International Congress on Big Data (BigDataCongress), pp. 111120, Milan, Italy, July 2019.
[7] E. Triantaphyllou, Multi-Criteria Decision-Making Methods: A Comparative Study, Kluwer, Norwell, MA, USA, 2000.

[8] T. Hassan, P. A. Shah, and F. A. Khan, "Fusion of motivational strategies with recommender system innovative framework for digital repositories," in Proceedings of the 2016 International Conference on Computing, Electronic and Electrical Engineering (ICE Cube), pp. 32-36, Quetta, Pakistan, April 2016.

[9] A. S. Ismail and H. Al-Feel, "Digital library recommender system on hadoop," in Proceedings of the 2015 IEEE Fourth Symposium on Network Cloud Computing and Applications (NCCA), pp. 111-114, Munich, Germany, June 2015.

[10] P. Jomsri, "FUCL mining technique for book recommender system in library service," Procedia Manufacturing, vol. 22, pp. 550-557, 2018.

[11] P. Knoth, L. Anastasiou, A. Charalampous et al., Towards Effective Research Recommender Systems for Repositories, pp. 26-30, Open Repositories, Brisbane, Australia, 2017.

[12] M. Borovič, M. Ferme, J. Brezovnik, S. Majninger, K. Kac, and M. Ojsteršek, "Document recommendations and feedback collection analysis within the slovenian open-access infrastructure," Information, vol. 11, no. 11, Article ID 497, 2020.

[13] M. Gupta, A. Thakkar, Aashish, V. Gupta, and D. P. S. Rathore, "Movie recommender system using collaborative filtering," in Proceedings of the 2020 International Conference on Electronics and Sustainable Communication Systems (ICESC), pp. 415-420, Coimbatore, India, July 2020.

[14] R. Singla, S. Gupta, A. Gupta, and D. K. Vishwakarma, "FLEX: a content based movie recommender," in Proceedings of the 2020 International Conference for Emerging Technology (INCET), pp. 1-4, Belgaum, India, June 2020.

[15] C. Troussas, A. Krouska, and M. Virvou, "Multi-algorithmic techniques and a hybrid model for increasing the efficiency of recommender systems," in Proceedings of the 2018 IEEE 30th International Conference on Tools with Artificial Intelligence (ICTAI), pp. 184-188, Volos, Greece, Novenber 2018.

[16] K. Kabassi and A. Martinis, "Studies for designing stereotypes in a mobile recommendation system for environmental awareness," in Proceedings of the 15th IEEE International Workshop on Semantic and Social Media Adaptation and Personalization (SMA), pp. 1-6, Zakynthos, Greece, October 2020.

[17] K. Chrysafiadi, C. Troussas, and M. Virvou, "A framework for creating automated online adaptive tests using multiple-criteria decision analysis," in Proceedings of the 2018 IEEE International Conference on Systems, Man, and Cybernetics (SMC), pp. 226-231, Miyazaki, Japan, October 2018.

[18] J. L. D. Leal, C. Rodrigo, and A. Garcia-Serrano, "Towards a recommender system for digital resources based on social assessments: experiment in an educational context," in Proceedings of the 2014 9th Iberian Conference on Information Systems and Technologies (CISTI), pp. 1-6, Barcelona, Spain, June 2014.

[19] H. Slimani, N. El Faddouli, R. Benslimane, and S. Bennani, "Personalized search and recommendation in a digital educational resources repository: the case of ORI-OAI," in Proceedings of the 2016 4th IEEE International Colloquium on Information Science and Technology (CiSt), pp. 541-546, Tangier, Morocco, October 2016.

[20] K. Dahdouh, L. Oughdir, A. Dakkak, and A. Ibriz, "Smart courses recommender system for online learning platform," in Proceedings of the 2018 IEEE 5th International Congress on Information Science and Technology (CiSt), pp. 328-333, Marrakech, Morocco, October 2018. 
[21] T. Wongkhamchan, A. Namvong, and C. Surawanitkun, "Personalized recommender system using a social network based collaborative filtering technique," in Proceedings of the 2019 16th IEEE International Conference on Electrical Engineering/Electronics, Computer, Telecommunications and Information Technology (ECTI-CON), pp. 846-849, Pattaya, Thailand, July 2019.

[22] A. Mughaid, I. Obeidat, B. Hawashin, S. AlZu'bi, and D. Aqel, "A smart geo-location job recommender system based on social media posts," in Proceedings of the 2019 Sixth IEEE International Conference on Social Networks Analysis, Management and Security (SNAMS), pp. 505-510, Granada, Spain, October 2019.

[23] X. Yang, M. Dong, X. Chen, and K. Ota, "Recommender system-based diffusion inferring for open social networks," IEEE Transactions on Computational Social Systems, vol. 7, no. 1, pp. 24-34, 2020.

[24] B. Liang, B. Xu, X. Wu et al., "A community-based collaborative filtering method for social recommender systems," in Proceedings of the 2019 IEEE International Conference on Web Services (ICWS), pp. 159-162, Milan, Italy, July 2019.

[25] S.-M. Choi, K. Jang, T.-D. Lee, A. Khreishah, and W. Noh, "Alleviating item-side cold-start problems in recommender systems using weak supervision," IEEE Access, vol. 8, pp. 167747-167756, 2020.

[26] K. El Handri and A. Idrissi, "Parallelization of $\$\{$ Top_ $\{k\}\} \$$ algorithm through a new hybrid recommendation system for big data in spark cloud computing framework," IEEE Systems Journal, pp. 1-11, 2020.

[27] S. Fomba, P. Zarate, M. Kilgour, G. Camilleri, J. Konate, and F. Tangara, "A recommender system based on multi-criteria aggregation," International Journal of Decision Support System Technology, vol. 9, no. 4, pp. 1-15, 2017.

[28] S. Vairamuthu, A. Anthoniraj, S. Margret Anouncia, and U. K. Wiil, "User interface design recommendations through multi-criteria decision analysis," in Knowledge Computing and its Applications: Knowledge Manipulation and Processing Techniques, S. M. Anouncia and U. K. Wiil, Eds., Springer, Berlin, Germany, pp. 269-293, 2018.

[29] V. Gupta and S. Pandey, "Recommender systems for digital libraries: a review of concepts and concerns," Library Philosophy and Practice, Article ID 2417, 2019.

[30] F. Cacheda, V. Carneiro, D. Fernández, and V. Formoso, "Comparison of collaborative filtering algorithms," ACM Transactions on the Web, vol. 5, no. 1, pp. 1-33, 2011.

[31] C. Troussas, A. Krouska, and C. Sgouropoulou, "Improving learner-computer interaction through intelligent learning material delivery using instructional design modeling," Entropy, vol. 23, no. 6, Article ID 668, 2021. 